\title{
Editorial
}

\section{Can maternal variables alone determine immunization (Drossark coverage?}

One of the major determinants of under-five mortality reduction in India has been the introduction of effective universal immunization program (UIP). It was envisaged that by the year $1990,85 \%$ of the infants would be covered. However, after initial success, there has been a dismal decline in the UIP coverage across most states in India due to may factors. ${ }^{1}$ Since the reasons for low UIP coverage could vary by georgraphic area, among urban and rural population, across income quintiles etc., there is a continued need to evaluate context specific associates with the aim to improve immunization coverage across the country.

Awasthi et al. ${ }^{2}$ have assessed the maternal determinants of immunization in urban slums, an area where focused research is needed, since the number of slums and slum dwellers in India is increasing exponentially. They used a modified WHO EPI cluster sampling method while conducting a crosssectional survey of 200 slums in Varanasi, India, in 2009. House-to-house visits were done to collect information on 384 children using pretested questionnaire. They report that only $57.03 \%$ children received the complete recommended immunization schedule under UIP, with vaccine coverage dipping from the first to the last recommended dose. In multinomial logistic regression analysis, the statistically significant determinants of complete immunization were maternal age younger than 30 years, those with a maximum of 2 children, employed and educated beyond post-primary.

Awasthi et al. ${ }^{2}$ have meticulously documented their methods and results. Their finding that younger mothers are more likely to complete UIP for their infants can be leveraged upon to improve the coverage rates in subsequent cohorts of infants systematically.

However, researchers have reported gender as an independent determinant of immunization in India. ${ }^{3}$ Gender, interestingly, had a univariate association with UIP coverage in the current study but was not picked up during the logistic regression analysis. This raises the issue of whether bias and confounding were dealt adequately in the current study. This is pertinent as the gender ratio of children of respondents was $>1$ for male when compared to females.
Likewise, variables which could have programmatic implications, such as awareness of type of vaccine, benefits of immunization and side effects of vaccine had univariate association only with complete UIP coverage. Could these variables be surrogate markers of maternal education? Would raising maternal awareness levels for immunization benefits increase coverage rates independent of maternal education? This remains unanswered and needs further research and perhaps even secondary data analysis by the current authors. The maternal determinants must be evaluated with all other variables ${ }^{1}$ to establish their relative potential for contributing to improved immunization rates in India on an urgent basis.

\section{RE F E R E N C E S}

1. Vashishtha VM, Kumar P. 50 years of immunization in India: progress and future. Indian Pediatr. 2013;50:111-118.

2. Awasthi A, Pandey CM, Singh U, Kumar S, Singh TB. Maternal determinants of immunization status of children aged 12-23 months in urban slums of Varanasi, India. Clin Epid Glob Health. 2015;3:113-119.

3. Pande R. Selective gender differences in childhood nutrition and immunization in rural India: the role of siblings.

Demography. 2003;40:395-418.

Shally Awasthi* Professor, Department of Pediatrics, King George's Medical University, Lucknow, India

Jai Vir Singh

Professor and Head, Department of Community Medicine and Public Health, King George's Medical University, Lucknow, India

${ }^{*}$ Corresponding author E-mail address: shally07@gmail.com (S. Awasthi) http://dx.doi.org/10.1016/S2213-3984(15)00062-7 2213-3984/

(C) 2015 INDIACLEN. Published by Elsevier B.V. All rights reserved. 